Research Article

\title{
Application of Internet of Things Compressed Sensing and Information Interaction Technology in Intelligent Transportation Layout
}

\author{
Na Li $(\mathbb{D}$, Ze Wu $(\mathbb{D}$, and Zhongbiao Zhao \\ School of Electrical and Mechanical Engineering, Xuchang University, Xuchang 461000, China \\ Correspondence should be addressed to Na Li; ln@xcu.edu.cn
}

Received 8 March 2021; Revised 7 April 2021; Accepted 14 April 2021; Published 23 April 2021

Academic Editor: Yi-Zhang Jiang

Copyright (c) $2021 \mathrm{Na} \mathrm{Li} \mathrm{et} \mathrm{al.} \mathrm{This} \mathrm{is} \mathrm{an} \mathrm{open} \mathrm{access} \mathrm{article} \mathrm{distributed} \mathrm{under} \mathrm{the} \mathrm{Creative} \mathrm{Commons} \mathrm{Attribution} \mathrm{License,} \mathrm{which}$ permits unrestricted use, distribution, and reproduction in any medium, provided the original work is properly cited.

\begin{abstract}
With the continuous development of economy, the number of motor vehicles has increased sharply, and urban traffic congestion has become more and more frequent. Urban traffic congestion has become one of the important reasons that hinder the continuous development of major cities. In order to better solve the problem of urban traffic congestion, it is more urgent to build urban intelligent transportation system. The technology related to Internet of things is becoming more and more mature, which has become a new idea of collecting traffic information, providing convenience for traffic, and injecting vitality into the study of intelligent transportation layout. The traffic system in the city or the main traffic intersection, through the traffic guidance layout road, provides the traffic instruction for the passenger on the highway network, enables the driver to choose the suitable driving road, can provide the travel guidance service for the driver, adjusts the flow distribution, and improves the traffic condition. This paper describes the research status of intelligent transportation layout and uses the basic theory of compressed sensing and information interaction technology to carry out data fusion and reconstruction algorithm in intelligent transportation. Finally, the application of compressed sensing in intelligent transportation layout is summarized to realize data collection in intelligent transportation network. In this paper, compressed sensing theory is applied to the data acquisition of intelligent transportation network to reduce the amount of data and improve the effective data acquisition. The reconstruction algorithm is used to realize the data reconstruction to ensure the accuracy and stability of signal reconstruction and the accuracy of network transmission information.
\end{abstract}

\section{Introduction}

With the rapid development of domestic economy and the continuous improvement of people's income, China's urbanization level has reached an unprecedented height. With the rapid expansion of urban area and the rising of housing prices, urban population is constantly moving to the suburbs. In order to improve the efficiency of travel and shorten the consumption of time on the way to work, more and more families use cars as a necessary means of travel, resulting in a sharp increase in the number of urban motor vehicles [1]. Although the number of urban traffic roads has increased and the carrying capacity of existing urban traffic roads has also been improved, it still cannot meet the demand for motor vehicles for roads in the same period. Therefore, urban traffic roads are becoming more and more crowded. The phenomenon of urban traffic congestion has attracted the attention of governments all over the world and has become an urgent problem for traffic managers in major cities [2]. Urban traffic congestion has a serious impact on the daily travel of urban residents and has become one of the main factors hindering the further improvement of the level of urbanization. Traffic congestion not only reduces the traffic capacity of the road but also shortens the service life of the road and greatly increases the traffic delay time of urban residents [3]. In addition, urban traffic congestion will lead to a series of problems, which has brought great threat to the life and property of the country and the people and has become the focus of public opinion. Aiming at a series of major problems caused by urban traffic congestion, 
governments around the world deepen cooperation in this field [4]. At the same time, a large number of researchers have also invested in the research of urban traffic congestion. Intelligent transportation layout has greatly expanded people's traditional understanding of urban transportation and opened up new ideas for the future planning of urban transportation. With the development of its research, it has been widely considered as one of the best ways to solve the problem of urban traffic congestion. In order to solve the problem of urban traffic congestion, the governments of various countries also continue to increase the investment in infrastructure construction, so as to prepare for the comprehensive completion of the intelligent transportation system [5].

The construction of intelligent transportation system has become the only way for the sustainable development of urban transportation. Intelligent transportation will be the future development direction of transportation. It will make the traditional transportation mode more intelligent, safer, energy-saving, and efficient through high technology. Internet of things is an important part of the new generation of information technology; through radio frequency identification, global positioning system, and other types of information sensing equipment, according to the agreed protocol, any object is connected to the Internet for information exchange and communication. With the continuous development of Internet of things technology, it also injects new power into the further development and improvement of its. In view of the serious harm of urban traffic congestion to urban development, this paper proposes an intelligent transportation layout based on Internet of things compressed sensing and information interaction technology to realize the traffic layout estimation method based on compressed sensing. Experimental results show that the sparse coding method can achieve more effective sparse representation of traffic layout information at adjacent times and can obtain lower estimation error. In order to further reduce the estimation error of compressed sensing method for urban traffic layout, this paper proposes a sparse representation matrix based on Gaussian kernel function by combining the road network topology and introducing the Gaussian joint distribution model, so as to improve the sparsity performance of traffic layout information, and proposes a method to estimate the traffic layout by using part of the detection vehicles; this method constructs a sparse representation matrix; it analyses whether the sparse representation matrix and measurement matrix constructed by this method meet the dual incoherence principle in compressed sensing theory. The simulation results of real data show that this method can reduce the reconstruction error of traffic layout information and achieve more accurate estimation of urban traffic layout.

This paper mainly describes the content of the paper from six parts, and the specific arrangement of each chapter is summarized as follows: the first section is the introduction, which mainly introduces the specific topic background and research significance of the paper, analyses the research status of compressed sensing theory and information interaction technology in the application of Internet of things at home and abroad, and summarizes the main research content and paper structure arrangement of this paper. The second section discusses the related work. The third section introduces the Internet of things compressed sensing model and information interaction technology and analyses and verifies the usability, efficiency, flexibility, and security of the proposed model from the theoretical and experimental aspects. The fourth section discusses the application of compressed sensing and information interaction technology based on the Internet of things in intelligent transportation, from the aspects of compressed sensing data collection and application effectiveness. In Section 5, the performance of this method is verified by simulation. The sixth section is the summary and prospect of this paper.

\section{Related Work}

As an important basis for the implementation of traffic road planning, traffic layout makes a large number of scholars invest in the study of traffic layout estimation methods [6]. The main research methods can be divided into three categories: one is based on data fusion; the other is based on traffic information matching and tracking; the third is based on traffic dynamics. The technology related to Internet of things is becoming more and more mature, which has become a new idea of collecting traffic information, providing convenience for traffic, and injecting vitality into the study of intelligent transportation layout [7]. Intelligent transportation will be the future development direction of transportation. It will make the traditional transportation mode more intelligent, safer, energy-saving, and efficient through high technology [8]. In recent years, the application of compressed sensing in intelligent transportation layout has received extensive attention and development.

A large number of data simulation experiments have been carried out in the research of road traffic layout estimation methods, and the advantages and disadvantages of multiple regression, fuzzy reasoning, neural network, and speed integral on the traffic layout estimation model are analysed [9]. Some researches propose data fusion traffic estimation method based on robust Kalman filter algorithm. This method mainly uses the existing road GPS data information to estimate the road traffic layout with missing GPS data, which improves the integrity of road traffic layout estimation. Some scholars use Bayesian estimation, fuzzy logic, and other data fusion methods to establish a multiheterogeneous traffic information three-level fusion system and obtain high-precision and high-reliability traffic layout data [10]. Other studies have proposed real-time traffic layout estimation method based on floating vehicle data. Its main work is map matching processing, road section division method, and minimum sample size research on GPS data of service vehicles. In this paper, a series of data sources such as special 
vehicle detector, vehicle tracking and positioning system, and road toll system are studied, and the traffic data preprocessing and multidata fusion traffic layout monitoring method using multineural network are proposed [11].

According to the method of estimating road flow and density with detection data, through a large number of simulation studies, when the proportion of detection vehicles is $3.5 \%$ and $0.2 \%$, this method can accurately output traffic flow of 5 minutes and hours. In part of the study, we proposed a method to estimate the traffic layout of different roads by accurately estimating the average speed of road sections and obtained the estimation accuracy of $3 \%$ floating vehicles in congested flow which is equivalent to that of $30 \%$ floating vehicles in free flow through experiments. It is mentioned in some literature that the travel time of all road sections can be estimated by the historical data of road traffic, and the weight parameter mechanism is proposed to overcome the data sparsity [12]. In this paper, the idea of using probe vehicle to estimate urban traffic layout by using compressed sensing method is proposed. The method of traffic layout estimation based on the combination of probe vehicle and compressed sensing can reduce the cost of data collection [13]. With the in-depth study of traffic layout estimation methods by a large number of scholars, more and more traffic layout estimation methods have been proposed. Especially in the face of such a large amount of traffic flow data, compressed sensing method can greatly reduce the amount of data required for traffic layout estimation and can solve the problem of missing road data, which provides great technical support for the establishment of urban intelligent transportation system.

The application of compressed sensing theory has attracted the attention of many scholars in the field of medical imaging, astronomical observation, and speech compression. Aiming at the problem of a large amount of data information in wireless sensor network, some scholars use compressed sensing technology to observe the data of a single sensor node and carry out fusion processing in the process of data transmission, so as to realize the application of compressed sensing in wireless sensor network data collection $[14,15]$. Their research also shows that its application can significantly reduce the data storage and data transmission time; domestic scholars have also carried out research on compressed sensing theory. In the field of sensor networks, some people have studied efficient data transmission schemes in wireless sensor networks, others have applied compressed sensing to target positioning in wireless sensor networks, and others have applied compressed sensing to reduce low power consumption in body area networks. According to the literature review, there are no reports about compressed sensing to deal with the interactive sensing of massive vehicle sensing information. Many famous universities, including MIT, Harvard University, Carnegie Mellon University, Chinese Academy of Sciences, and Tsinghua University, have set up research groups on the theory and application of compressed sensing. CS has rapidly become a hot research field in recent years, and related research results emerge endlessly, which has been widely used in cognitive radio medical imaging, signal processing, and target setting in many fields, such as bit, wireless communication, radar detection, channel coding, channel estimation, geological exploration, and satellite remote sensing image [16]. In the current situation, although the expansion of roads and the control of the increase in the number of vehicles can alleviate the traffic pressure, the utilization of land resources is limited after all. This way cannot solve the congestion phenomenon from the perspective of sustainable development. This paper describes the research status of intelligent transportation layout and uses the basic theory of compressed sensing and information interaction technology to carry out data fusion and reconstruction algorithm in intelligent transportation.

\section{Internet of Things Compressed Sensing and Information Interaction Technology}

3.1. Compressed Sensing of Internet of Things. If the coefficient of the signal $x$ in the basis vector has the following properties, it is said that the signal $x$ is compressible in the basis vector: the absolute values of the coefficients of $\mathrm{W}$ are arranged from large to small. Generally, if a signal contains redundancy, it is compressible. Therefore, for a node data field related to data, we can reasonably assume that the signal is compressible. The $\mathrm{n}$-dimensional vector represents the approximation matrix of the largest $m$ elements, the remaining elements are set to zero and similarly introduced to represent the approximation matrix of the smallest elements, and the remaining elements are set to zero.

$$
G=\varphi \varepsilon_{s}+\varphi \varepsilon_{e} .
$$

It is obtained by random sampling. We assume that the compressed sensing data is noise data, which is represented by $N$ and obeys the Gaussian distribution of zero means.

$$
G=\varphi \varepsilon_{s}+n,
$$

where $n$ is a Gaussian distribution with zero mean and $\sigma^{2}$ variance. So, we get Gaussian likelihood estimation.

$$
H\left(G \mid \varepsilon_{s}, \sigma^{2}\right)=\frac{e\left\|G-\varphi \varepsilon_{s}\right\| / \sigma^{2}}{\pi \sigma^{2}} .
$$

Considering the accuracy of the Gaussian density function, we can get the following results:

$$
H(\varphi \mid \alpha)=\prod_{i=1}^{T} T\left(\varphi_{i} \mid 0, \alpha_{i}\right)+\Gamma\left(\alpha_{i} \mid \tau, v\right) .
$$

Ignoring the influence of $\alpha$, the weight can be calculated by the following formula:

$$
H(\varphi \mid \tau, v)=\prod_{i=1}^{T} \int_{0}^{\infty} T\left(\varphi_{i} \mid 0, \alpha_{i}\right)+\Gamma\left(\alpha_{i} \mid \tau, v\right) d \alpha_{i} .
$$

Through the above analysis, the research of coefficient degree in compressed sensing is transformed into a linear regression problem. The prior constraint of this problem is sparse. The widely used sparse promotion algorithm is the Laplace density function. 


$$
H(\varphi \mid \lambda)=\prod_{i} \int H\left(\varphi_{i} \mid \gamma_{i}\right) H\left(\gamma_{i} \mid \lambda\right) d \gamma_{i}
$$

Compressed sensing can be used to construct classifiers. According to the theory of compressed sensing, the signal can be compressed, or the signal is sparse in a certain sensing field. The sparse signal can be projected into a low dimensional space. Through optimization, the high probability signal in the projection can be reconstructed into the original signal. The reconstruction signal contains a lot of information and effectively distinguishes the original signal; the principle of its main implementation is shown in Figure 1.

In signal compression, the signal is first transformed, such as discrete cosine transform or wavelet transform, then a few coefficients with larger absolute value are compressed and coded, and the zero or near zero coefficients are discarded [17]. The measured value is not the signal itself, but the projection value from high dimension to low dimension. From the mathematical point of view, each measured value is the combination function of each sample signal in the traditional sense; that is, a measured value contains a small amount of information of all sample signals [18]. By compressing the data, most of the sampled data is discarded, but the perception effect is not affected. For example, when a digital camera with millions of pixels is used to image the scene, a large amount of pixel information will be obtained, but only a small amount of information will be stored and transmitted after compression coding, and finally, the original image will be reconstructed through the corresponding decompression algorithm. If the signal itself is compressible, can we directly obtain its compressed representation (i.e., compressed data) so as to omit the sampling of a large amount of redundant information? Cand proved in 2006 that the original signal can be accurately reconstructed from some Fourier transform coefficients, which laid a theoretical foundation for compressed sensing. Then Cand é $s$ and Donohoe put forward the concept of compressed sensing on the basis of related research. The core idea is that sampling and compression coding occur in the same step. Firstly, the nonadaptive linear projection of the signal is collected, and then the original signal is reconstructed from the measured value according to the corresponding reconstruction algorithm. Modules of information interaction system were shown in Figure 2.

Through research, it has been known that as long as the signal is compressible or sparse in a certain transform domain, then the high-dimensional signal obtained from the transformation can be projected onto a low dimensional space by using an observation matrix not related to the transform base, and then the original signal can be reconstructed with high accuracy from these few projections by solving an optimization problem, which can prove such a projection value package. It contains enough information of reconstructed signal; in this theoretical framework, the sampling rate is mainly determined by the sparsity and noncorrelation of information in the signal, not by the bandwidth of the signal.

3.2. Information Interaction Technology. Information exchange of Internet of things is a process of information transmission, information sharing, and information exchange based on network system with many heterogeneous network nodes [19]. Through information exchange, each node of Internet of things can obtain the information of environment and other nodes intelligently and autonomously. Although the existing research work has carried out in-depth research on human-computer interaction theory of traditional information system and put forward a complete information exchange model interaction model, there is no mature theoretical system for information interaction of Internet of things [20]. Through in-depth study of humancomputer interaction process of large-scale information system, the model thinks that information interaction is completed by the interaction among three basic objects: user, system, and content. The fundamental purpose of user using information system is to use the content of the system, but the user should obtain the information successfully content and must use the function provided by the system to complete the corresponding system operation, and the content is the system as a carrier for different applications to display information. Based on the above information interaction model, combined with the characteristics of the Internet of things, this paper puts forward the information interaction model of the Internet of things as shown in Figure 3; the basic object of the model is composed of three parts: user, network, and content different from the meaning of user in the traditional information interaction model; the user here is a generalized user, including not only the traditional human-computer interaction user but also the sink node, cluster head node, routing node, and general network node. The information interaction system of the Internet of things refers to the perception network itself; that is, the whole system including information perception unit, operation and storage unit, and energy unit information space based on the Internet of things network system constitutes the content of information interaction, including various perception data of network nodes, network state information, and high-level semantic and event information that users are interested in sink node obtains the interaction process of node perception information through the perception network [21].

\section{Application of Compressed Sensing and Information Interaction Technology Based on Internet of Things in Intelligent Transportation}

4.1. Acquisition of Compressed Sensing Data. In the vehicle road coordination system, the vehicle's microprocessor is mainly used to obtain on-board information and analyse and process the underlying information required by various applications in the vehicle road coordination system and put in various intelligent algorithms to comprehensively process all kinds of information, and send the corresponding information to the surrounding vehicles through the vehicle self-organizing network according to the needs. The collection of various heterogeneous sensors on the vehicle makes the vehicle have the ability to collect and identify environmental information, such as the condition 


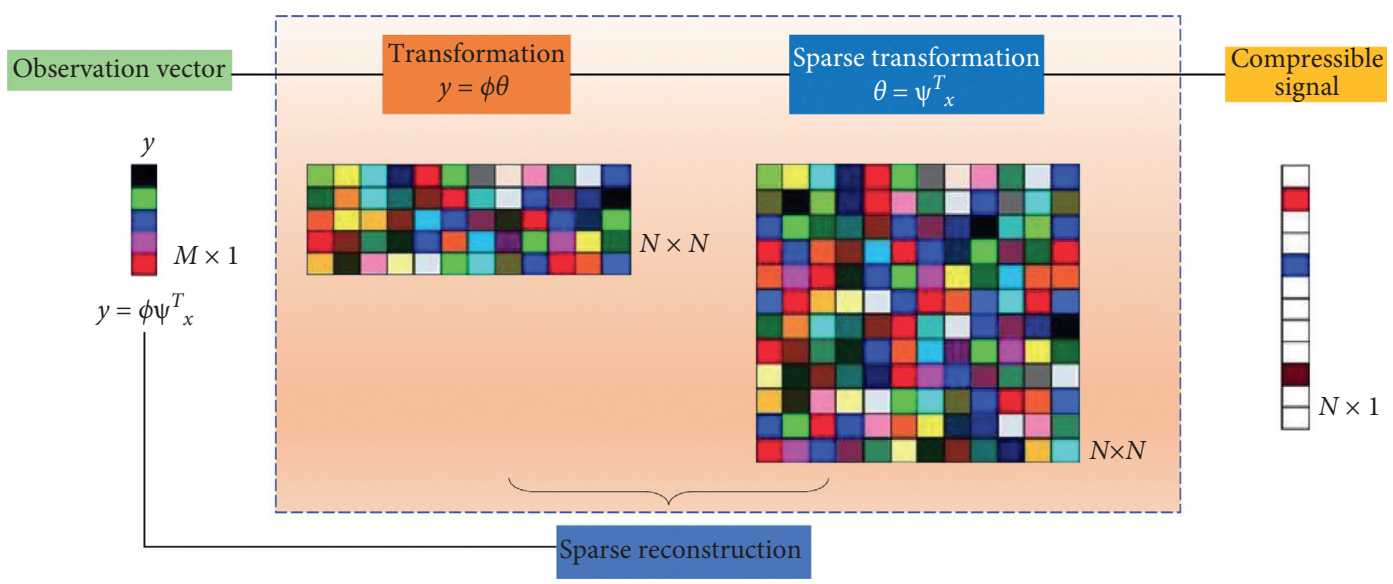

Figure 1: Schematic diagram of compressed sensing principle.

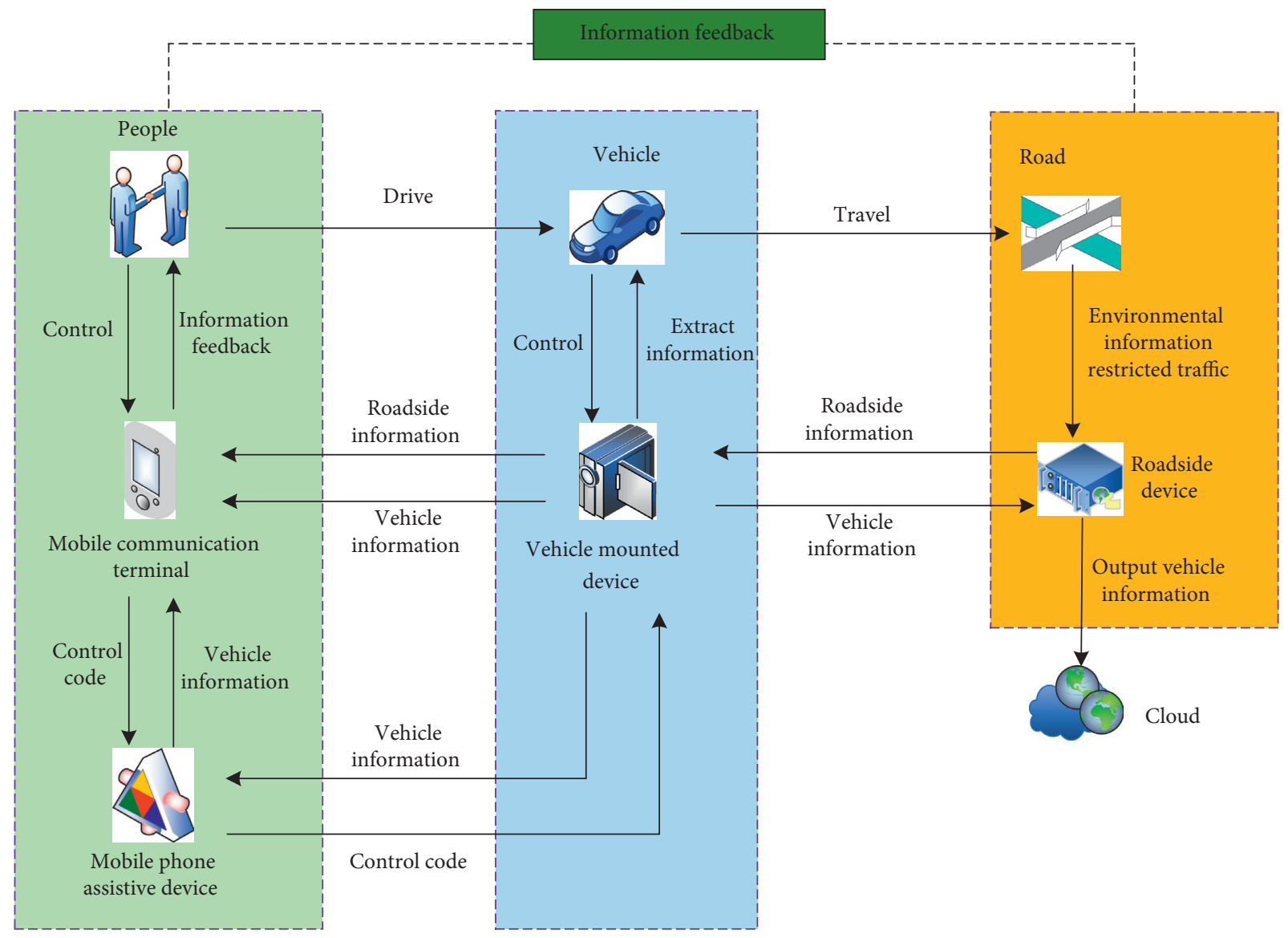

Figure 2: Modules of information interaction system.

acquisition of the vehicle running process [22]. With the use of GPS and the installation of acceleration sensor, gyroscope, steering wheel angle sensor, and other types of sensing equipment, the high-precision vehicle motion state information can be obtained. The measurement of vehicle relative positioning information is also detected by the on-board sensors, and distance sensors and vision sensors are mainly used to measure the relative positioning information of the vehicle [23]. The information of the driving environment around the vehicle is mainly obtained by radar, CCD vision sensor, infrared video sensor, and other types of equipment. Therefore, the ability of vehicle information acquisition under the vehicle road coordination system is greatly increased, which expands the original collection mode and collection range of traffic information. At the same time, the amount of data to be processed is also increasing at an amazing speed, which increases the information processing of the vehicle sensor network and the transmission burden of the vehicle sensor node [24]. As a vehicle sensor node, how to get the least required information in time and reduce 


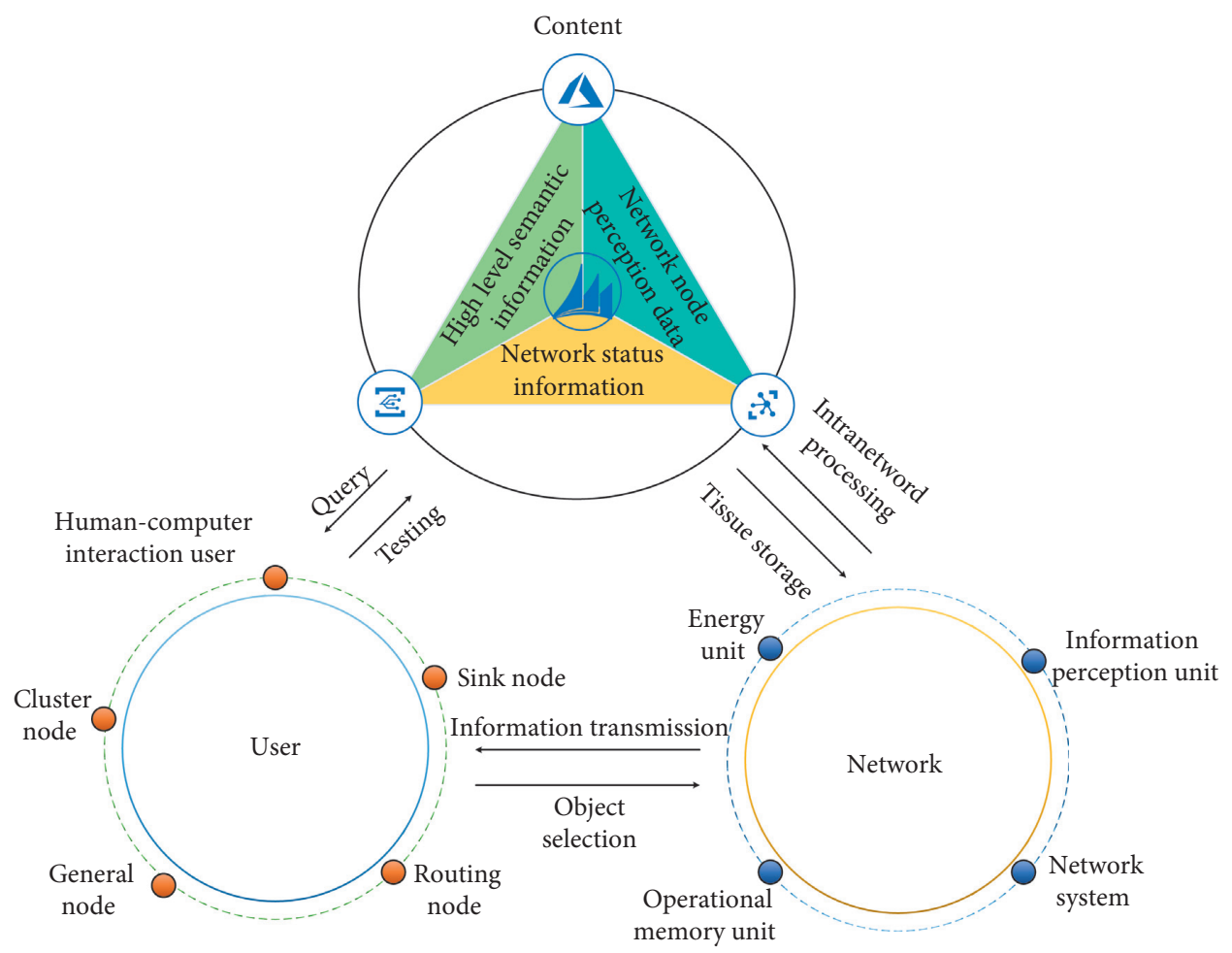

FIgURE 3: Information interaction model of Internet of things.

the cost in order to reduce the waste of broadband caused by unnecessary information collection and transmission, this paper proposes a data compression sensing acquisition method for vehicle sensor network.

The sparse representation of signal is to seek as few basic functions as possible in the transform domain. Fourier transform and wavelet transform are typical signal basis representation methods. The basic idea of wavelet transform is to use a set of wavelets or basis functions to represent a function or signal through scaling and translation. When the signal is represented by wavelet basis, the statistical characteristics of the signal can still be maintained under different scales or compression ratios [25]. Therefore, the original signal detected by vehicle sensor node can be transformed into wavelet domain to meet the prior conditions of compressed sensing, which is also a feasible method to reduce the redundancy of vehicle sensor information. After wavelet transform, the low-frequency part represents the outline of the signal, and the high-frequency part represents the details of the signal [26]. After decomposition, the wavelet coefficients are sorted according to the absolute value, and a certain proportion of the coefficients with relatively large absolute value are retained to obtain the sparse representation of the signal [27]. According to the analysis of vehicle cooperation method, vehicle acceleration information and speed information are important parameters to control vehicle cooperative driving. Figure 4 shows 300 high-frequency sampling points within 5 minutes of vehicle driving speed. It can be seen from Figure 4 that the condition of strict sparse compressed sensing is not satisfied. Compared with the standard Fourier transform, the wavelet

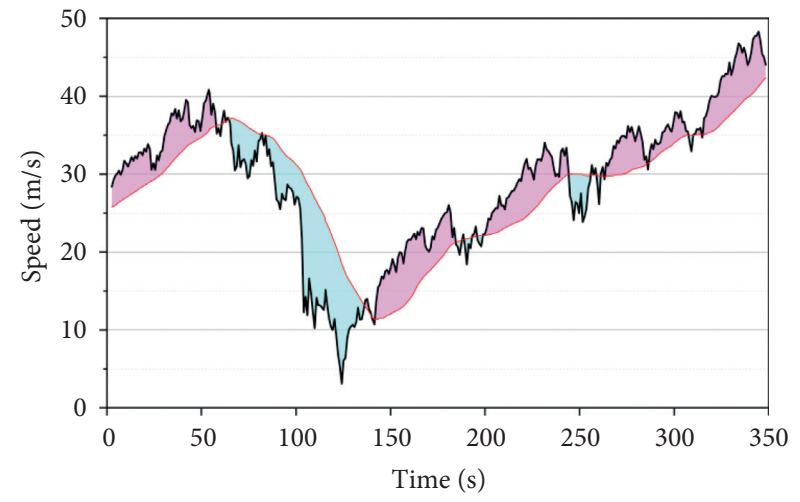

Figure 4: Curve of change value of vehicle sensing speed.

functions used in wavelet transform are not unique and diverse. Daubechies function in wavelet theory is a wavelet function constructed by Ingrid Daubechies, a famous wavelet analyst in the world. Except Hear wavelet, other wavelets have no explicit expression, but the square modulus of the transform function is very clear.

For the speed perception of vehicle, the wavelet transform method is used to transform the speed signal, and the corresponding orthogonal transform matrix is obtained. The unified concept of multiresolution analysis in wavelet theory establishes the construction methods of various wavelet bases. Through this analysis method, the orthogonal basis can be constructed for the speed signal perceived by the vehicle. Because of the FIR filter bank corresponding to the tightly supported wavelet base, the construction of wavelet 
base is easier to realize. A vehicle sensor signal is analysed by multiresolution method. The scale function $\mathrm{K}$ is selected and $u$ is established as a set of orthogonal scale functions. Through the above steps, the wavelet orthogonal basis vector $g$ of vehicle speed information is obtained, which satisfies the sparse condition of compressed sensing.

It can be seen from Figure 5 that the speed values of some continuous high-frequency sampling points are the same, and most of the change values of the vehicle sensing acceleration value should be 0 . The high-frequency sampling of vehicle sensing acceleration is shown in Figure 6. Among the 300 sampling points, 100 nonzero points are randomly distributed in the 300 sampling points. The vehicle sensing acceleration signal is strictly sparse, which fully adapts to the compressed sensing theory, so the sparse basis is the unitary matrix.

\subsection{Analysis of Application Effectiveness of Compressed} Sensing. In order to verify the application effectiveness of compressed sensing theory of vehicle sensing information, MATLAB is used to simulate the compressed sensing of vehicle sensing information. The MSE of the reconstructed results of vehicle sensing speed and acceleration under different measurement times $m$ is displayed. Due to the random uncertainty of Gaussian matrix, the mean square error is obtained by averaging the error obtained from 10 times of simulation for each observation.

For the vehicle sensing velocity and acceleration information in a certain period of time, the observation value $m$ is larger; that is, the more high-frequency sampling points of vehicle sensing data are, the more accurate reconstruction ratio is obtained by simulation. When the $m$ value of random observation is greater than 150, the reconstructed information error is stable. At this time, 150 sampling points were randomly selected from 300 high-frequency sampling points in 5 minutes. The result of information reconstruction is shown in Figure 6. The absolute error of the reconstructed value of a single sampling point can be obtained. The compressed sensing method can use a small amount of data to get more accurate reconstruction and recovery. The reconstruction and recovery error are small, and some error values are close to 0 . It is feasible to apply this method to the collection of vehicles sensing data.

\section{Analysis of Simulation Experiment}

5.1. Validation Analysis under Different Vehicle Density Scenarios. The sparsity of vehicle nodes in its layout is directly related to the stability of its layout state, the connectivity of its routing, and the competitiveness of its channel access. In order to explore the impact of different access protocols and different vehicle density on its layout performance, two scenarios with different vehicle density are designed in this simulation experiment. According to the design of the simulation experiment, the message delay and throughput are simulated. Through the simulation results, it can be concluded that, under the IOT compressed sensing and information interaction technology, the performance of message delay and throughput is better than that of traditional technology. When the simulation system has 100 vehicle nodes and 50 vehicle nodes, in terms of delay, the IOT compressed sensing and information interaction technology reduce $8.2 \%$ and $8.3 \%$, respectively, compared with the traditional technology; in terms of throughput, the IOT compressed sensing and information interaction technology increase $8.1 \%$ and $12.2 \%$, respectively, compared with the traditional technology.

The reason for the outstanding effect of Internet of things compressed sensing and information interaction technology is that the initial value of the competition window is set more reasonably after the message is sent successfully. When the number of vehicles is small, when the backoff algorithm is implemented, the initial value of backoff counter is small and the backoff time is short; when the number of vehicles is increased, the initial value of backoff counter is large and the backoff time is long. This method can adaptively adjust the initial value, change the value of the backoff counter according to the vehicle density, and reduce the possibility of channel transmission conflict as much as possible. Through the simulation, it is found that the different number of vehicle nodes also has an impact on the performance parameters of intelligent transportation layout. Therefore, this experiment designs an intelligent transportation layout environment with different number of vehicle nodes. The simulation results in Figure 7 show that, with the increase of the number of vehicle nodes, there will be a relatively good peak of its layout performance. This is because when the number of vehicles is small, the path to the destination node is relatively small, and the amount of information interaction data is relatively small, so the delay may be large, and the throughput is not high; with the increase of the number of vehicles, the routing establishment is more perfect, and the information interaction process makes full use of the intelligent transportation layout resources, so that the performance of message delay and throughput is the best. When the number of vehicles continues to increase, the resources of its layout will be relatively congested, resulting in the deterioration of its performance index again.

\subsection{Validation Analysis of Simultaneous Interpreting Sce-} narios with Different Transmission Distances. In vehicle road cooperative system, the transmission distance of vehicle node is its communication radius. The transmission distance is directly related to the hop number of node information transmission and the number of neighbour nodes and also directly affects the strength of channel competition between different nodes. This simulation experiment compares the impact of optimized IOT compressed sensing and information interaction technology and traditional technology on the system communication performance under two different communication transmission distances of $100 \mathrm{~m}$ and $250 \mathrm{~m}$. $250 \mathrm{~m}$ is the standard case. In this simulation experiment, the simulation results for message delay and throughput are $100 \mathrm{~m}$ and $250 \mathrm{~m}$, respectively. When the transmission distance of vehicle nodes is $100 \mathrm{~m}$ and $250 \mathrm{~m}$, respectively, in terms of delay, the Internet of things compressed sensing and information interaction technology protocols reduce 


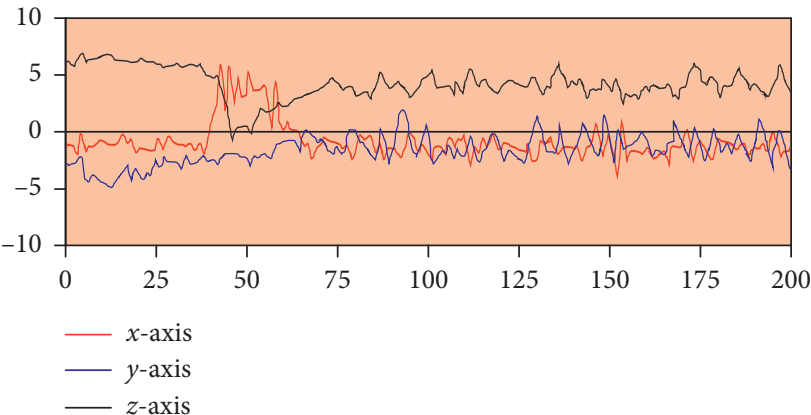

(a)

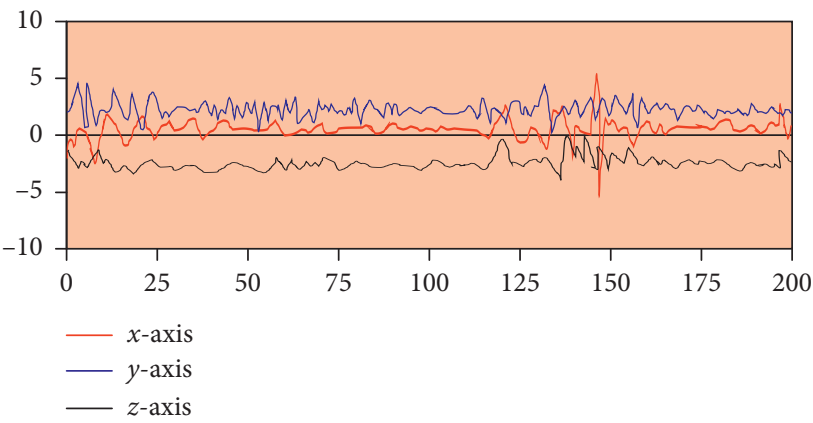

(c)

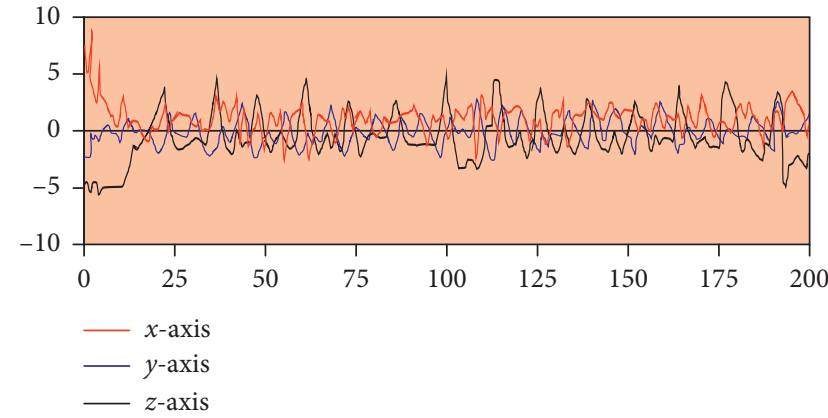

(b)

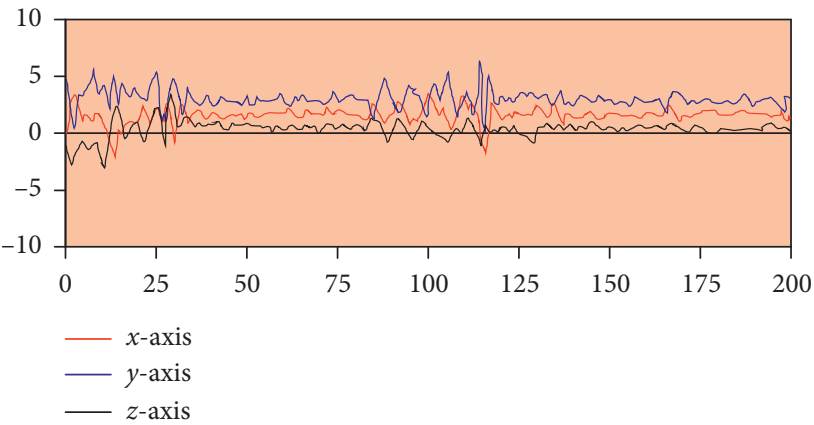

(d)

Figure 5: Decomposition of vehicle sensing speed.
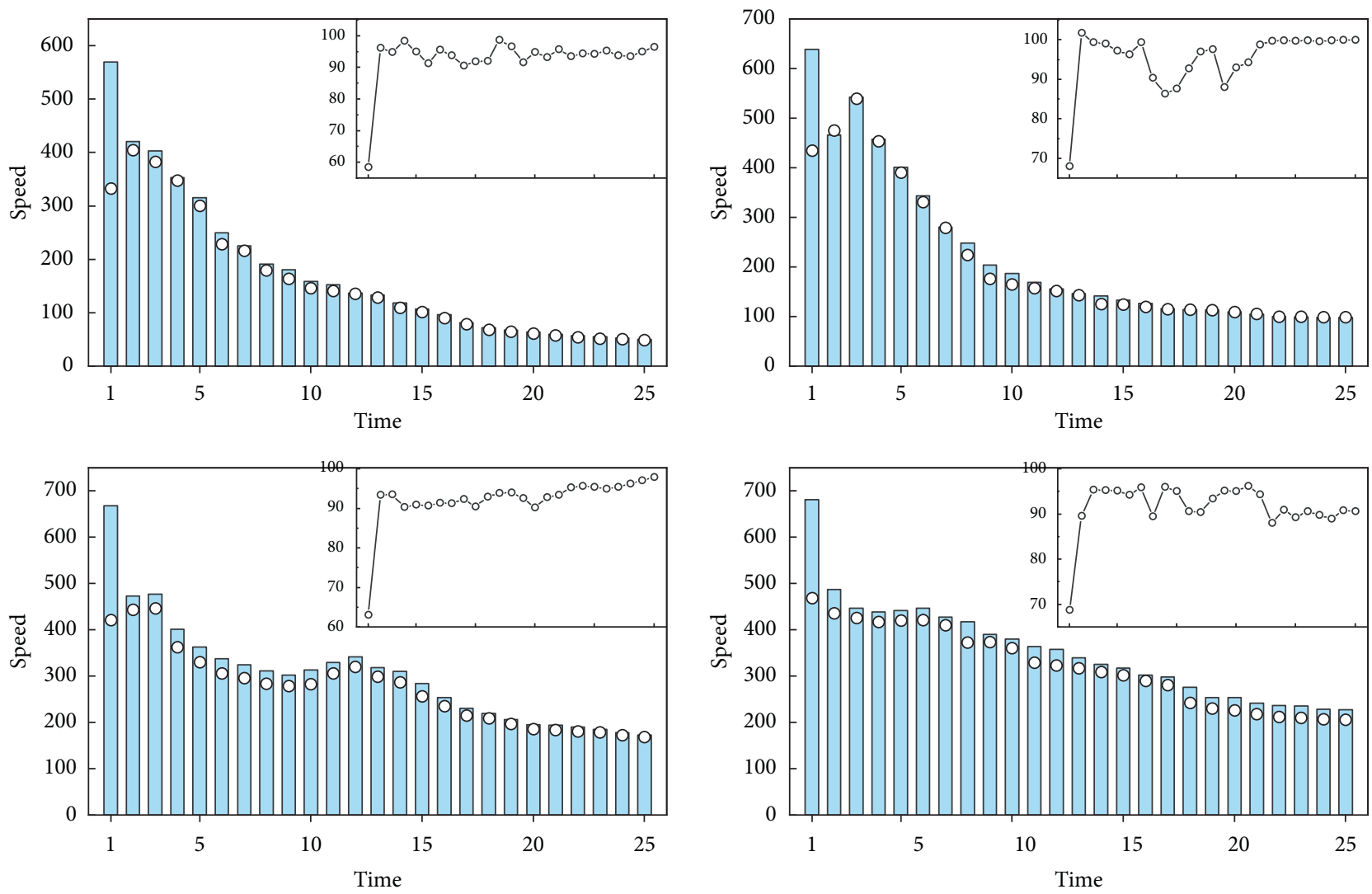

Figure 6: Compressed sensing reconstruction of vehicle sensing speed. 


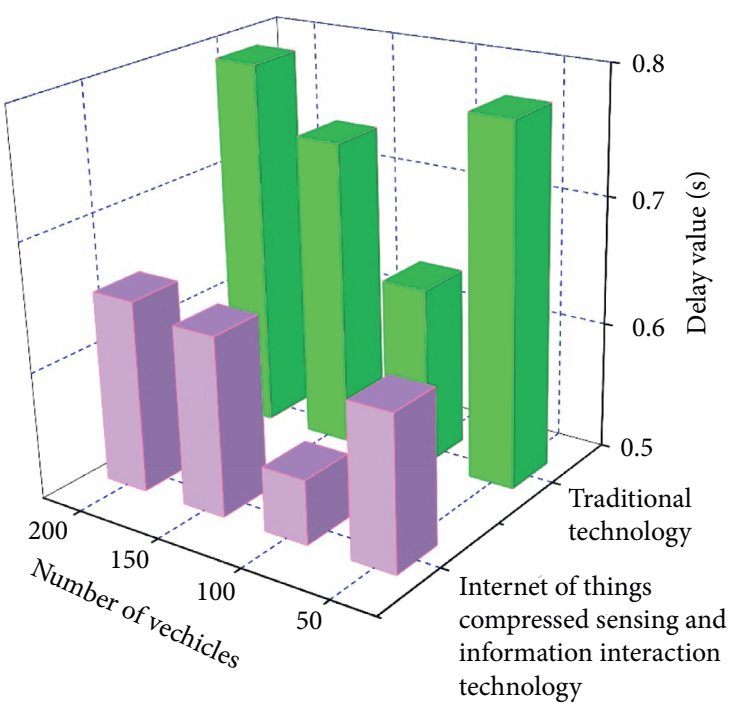

(a)

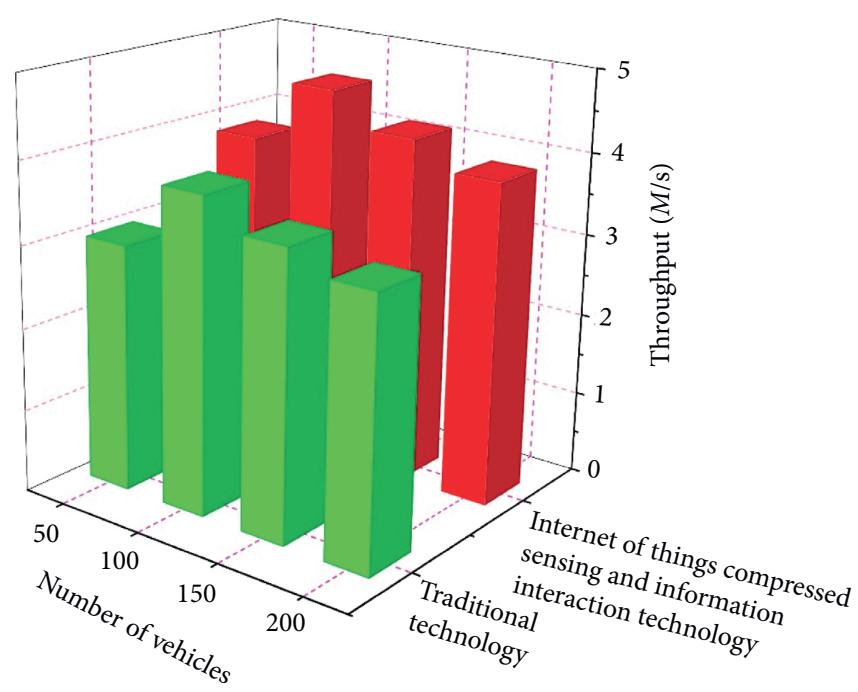

(b)

FIgURE 7: Simulation results of different vehicle density. (a) Comparison of time delay. (b) Comparison of throughput.

$7.6 \%$ and $8.2 \%$, respectively, compared with the traditional technology protocols, and in terms of throughput, the Internet of things compressed sensing and information interaction technology increase $14.1 \%$ and $8.1 \%$, respectively, compared with the traditional technology protocols.

This experiment simulates the communication intelligent transportation layout in the case of multiple transmission distances, and the simulation results are shown in Figure 8. With the increase of transmission distance, the performance of its layout changes in a " $U$ " shape. The reason for this phenomenon is that when the transmission distance is short and the number of transmission hops increases, the message delay is bound to be large and the throughput is not large. When the transmission distance is very large, the number of nodes communicating at the same time will decrease rapidly, the utilization rate of intelligent transportation layout resources will decrease, and the messages cannot be sent out in time, resulting in the increase of transmission delay and the decrease of intelligent transportation layout throughput. When the node transmission distance is relatively small, the probability of collision between nodes increases, resulting in an increase in the number of retransmissions; when the node transmission distance is relatively large, the number of transmission hops from the source node to the destination node increases, which takes up a long time of intelligent transportation layout resources, and will inevitably cause unnecessary transmission delay. Compared with the traditional technology, IOT compressed sensing and information interaction technology can sense the communication status and retransmission times of neighbour nodes and set reasonable backoff time slot.

5.3. Validation Analysis under Different Driving Speed Scenarios. The speed of vehicle nodes in vehicle road cooperative system has a decisive influence on the change rate of intelligent transportation layout topology. When the vehicle speed is extremely fast, it may lead to the misjudgement of hidden nodes and exposed nodes. This simulation experiment designs and compares the impact of IOT compressed sensing and information interaction technology and traditional technology on the communication performance of vehicle road cooperative system under two different driving speeds of $20 \mathrm{~km} / h$ and $40 \mathrm{~km} / h$ and sets $40 \mathrm{~km} / h$ as the standard case. The vehicle speed plays a decisive role in the change of its topology and has a great impact on its layout performance. In general, the compressed sensing and information interaction technology protocol of the Internet of things has improved in terms of delay and throughput compared with the traditional technology protocol. In the case of $20 \mathrm{~km} / h$ and $40 \mathrm{~km} / h$, the message delay is reduced by $8.5 \%$ and $8.2 \%$, respectively; in terms of throughput, it is increased by $16.6 \%$ and $8.1 \%$, respectively.

Through the simulation of the intelligent transportation layout of the vehicle road collaborative system under different vehicle speeds, it is found that the performance of the intelligent transportation layout is irregular, and there will not be peaks or troughs like the previous two experimental scenarios. The simulation results are shown in Figure 9. Speed is equivalent to random quantity in the vehicle road cooperative system. After all, compared with the radio transmission speed, the vehicle speed of $10 \mathrm{~km} / \mathrm{h}-70 \mathrm{~km} / h$ in this simulation system can be ignored.

In general, the effect of IOT compressed sensing and information interaction technology protocol is still better than that of traditional technology, mainly because IOT compressed sensing and information interaction technology protocol adaptively adjusts the competitive time window according to the number of retransmissions of nodes, reduces the time of nodes waiting for the channel when sending conflicts, and orderly uses the wireless intelligence in the traffic environment which can be used to arrange transportation resources. 


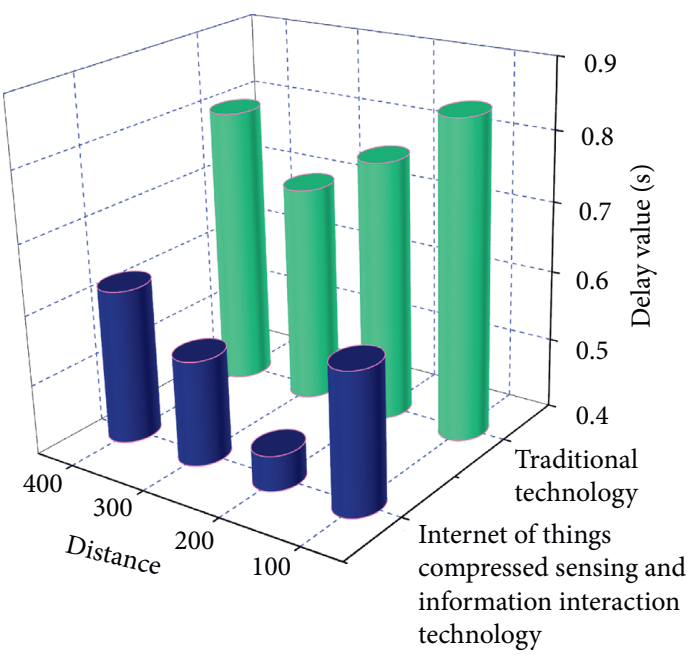

(a)

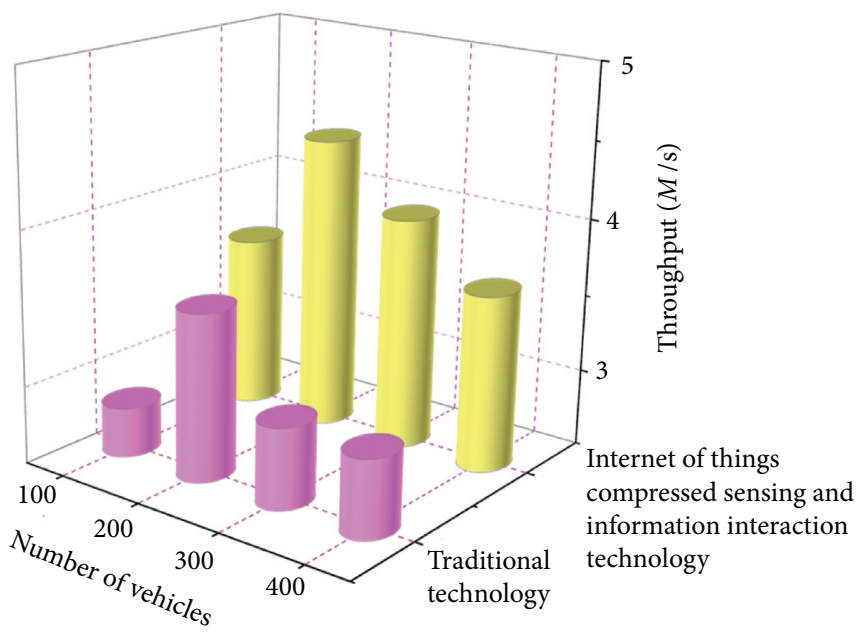

(b)

Figure 8: Simultaneous interpreting results of different transmission distances. (a) Comparison of time delay. (b) Comparison of throughput.

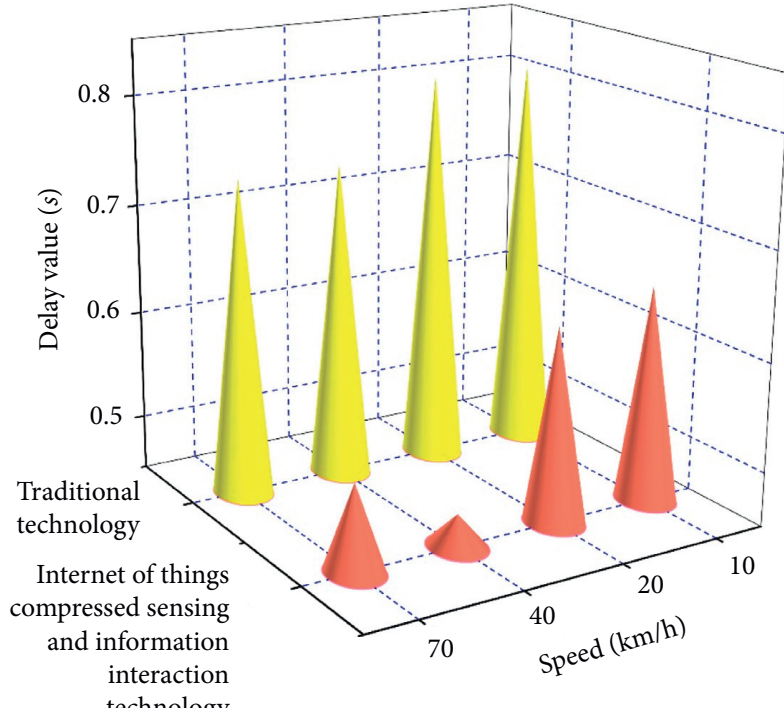

(a)

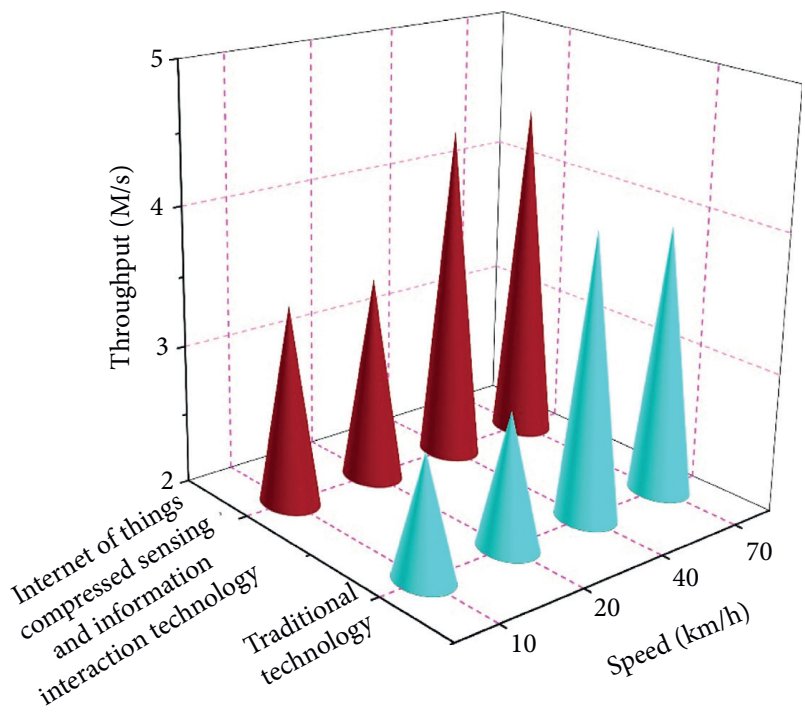

(b)

Figure 9: Simulation results of different vehicle speeds. (a) Comparison of time delay. (b) Comparison of throughput.

\section{Conclusion}

The rapid growth of car ownership has brought convenience to all aspects of people and led to frequent urban road congestion. Road congestion can have many negative effects. There are two main solutions, one is the traditional way of adding roads and controlling the increase in the number of vehicles, and the other is dredging. In the current situation, although the expansion of roads and the control of the increase in the number of vehicles can alleviate the traffic pressure, the utilization of land resources is limited after all. This way cannot solve the congestion phenomenon from the perspective of sustainable development. This paper describes the research status of intelligent transportation layout and uses the basic theory of compressed sensing and information interaction technology to carry out data fusion and reconstruction algorithm in intelligent transportation. Finally, the application of compressed sensing in intelligent transportation layout is summarized to realize data collection in intelligent transportation network. In this paper, compressed sensing theory is applied to the data acquisition of intelligent transportation network to reduce the amount of data and improve the effective data acquisition. The reconstruction algorithm is used to realize the data reconstruction to ensure the accuracy and stability of signal reconstruction and the accuracy of network transmission information. Finally, the simulation analysis is given. But at present, the feature dimension is very large, and the 
recognition process takes a long time, which cannot meet the real-time requirements. Further research is needed to guide the selection of feature dimension and the extraction of line features extracted by transformation and to solve the nonlinear pattern classification problem which is difficult to be solved by current compressed sensing methods. There will be more problems to be solved in the process of applying IOT compressed sensing and information interaction technology to the actual intelligent traffic layout.

\section{Data Availability}

Data sharing is not applicable to this article as no datasets were generated or analysed during the current study.

\section{Consent}

Informed consent was obtained from all individual participants included in the study references.

\section{Conflicts of Interest}

The authors declare that they have no conflicts of interest.

\section{References}

[1] Y. Gong and J. H. Liao, "Urban Intelligent Transportation big data platform based on blockchain technology and simulation case analysis," Highway Transportation Technology, vol. 36, no. 12, pp. 117-126, 2019.

[2] D. Wu and J. W. Hu, "Information perception and interaction technology of Internet of things," Computer Knowledge and Technology, vol. 13, no. 27, pp. 225-226, 2017.

[3] X. D. Wang, "Research on information perception and interaction technology of Internet of things," Journal of Shanxi Coal Management Cadre College, vol. 29, no. 1, pp. 204-205, 2016.

[4] Y. Wu, "Research on information perception and interaction technology of Internet of things," Journal of Chifeng University, vol. 32, no. 20, pp. 36-37, 2016.

[5] Y. D. Zhang and S. Z. Peng, "Construction of intelligent transportation system based on big data of internet of vehicles," Comprehensive Transportation, vol. 40, no. 11, pp. 25-29, 2018.

[6] Y. Q. Xia, C. Yan, X. Q. Wang, and X. H. Song, "Intelligent transportation information physical fusion cloud control system," Acta Automatic Sonica, vol. 45, no. 1, pp. 132-142, 2019.

[7] P. Zhang, "Application of internet of things technology in intelligent transportation," Science and Technology Communication, vol. 11, no. 4, pp. 158-159, 2019.

[8] J. Sun, "Cross integration of artificial intelligence and intelligent transportation," Information and Computer, vol. 11, no. 4, pp. 139-141, 2019.

[9] Y. S. Yang and X. Y. Li, "Research on intelligent transportation management and application based on big data technology," Journal of Chongqing Business University, vol. 36, no. 2, pp. 73-79, 2019.

[10] Z. Y. Luo, "Application of artificial intelligence technology in urban intelligent transportation," Communication World, vol. 26, no. 5, pp. 244-245, 2019.

[11] Z. Q. Yu and J. L. Li, "Design of intelligent transportation integrated information application platform based on big data," Journal of Henan Normal University, vol. 47, no. 4, pp. 37-41, 2019.

[12] Y. Y. Zhang, "Analysis on the development and application of artificial intelligence in intelligent transportation," Information Recording Materials, vol. 20, no. 5, pp. 105-106, 2019.

[13] W. Zhang, Y. Q. Wei, T. T. Liu, and S. L. Guan, "Application and platform construction of intelligent transportation system under the background of big data," Shanxi Architecture, vol. 45, no. 11, pp. 183-185, 2019.

[14] L. J. Zeng, "Research on urban intelligent transportation system design based on big data," Think Tank Era, vol. 35, no. 7, pp. 281-282, 2019.

[15] H. Mi and Y. Zheng, "Design and construction of intelligent transportation collaborative innovative training base based on intelligent transportation industry chain," Journal of Jinling University of Science and Technology, vol. 35, no. 3, pp. 26-31, 2019.

[16] Y. Tao, X. D. Yan, T. M. Wang, and M. Liu, "Development strategy of intelligent transportation system for future intelligent society," Science and Technology Guide, vol. 34, no. 7, pp. 48-53, 2016.

[17] L. B. Huang, "Review of urban intelligent transportation system construction at home and abroad," Urban Roads, Bridges and Flood Control, vol. 17, no. 5, pp. 40-45, 2016.

[18] Y. Wu, "Application of internet of things technology in intelligent transportation system architecture," Automation and Instrumentation, vol. 20, no. 6, pp. 131-132, 2016.

[19] F. Wang, D. S. Jiang, and H. M. Yue, "Research and development of intelligent traffic lights to effectively relieve urban traffic pressure," Journal of Changchun Institute of Technology, vol. 17, no. 3, pp. 101-104, 2016.

[20] Z. H. Wu and Y. He, "Making full use of intelligent transportation technology to improve road traffic safety," Traffic Information and Safety, vol. 33, no. 1, pp. 1-8, 2015.

[21] L. L. Bai and T. P. Han, "Application of big data in intelligent transportation system," Computer Knowledge and Technology, vol. 11, no. 10, pp. 204-206, 2015.

[22] H. P. Lu, Z. Y. Sun, and W. C. Qu, "Big data and its application in urban intelligent transportation system," Transportation System Engineering and Information, vol. 15, no. 5, pp. 45-52, 2015.

[23] H. F. Liu and P. H. Huang, "Intelligent transportation Internet of things technology and industrialization," Engineering Research Engineering in Interdisciplinary Perspective, vol. 6, no. 1, pp. 20-30, 2014.

[24] M. J. Jin, "Development status and prospect of intelligent transportation system technology in China," Traffic Information and Safety, vol. 30, no. 5, pp. 1-5, 2012.

[25] Z. S. Jiang, X. N. Tang, and X. H. Chen, "Architecture analysis of internet of vehicles and its application in intelligent transportation system," Internet of Things Technology, vol. 2, no. 11, pp. 39-41, 2012.

[26] J. F. Bai, "Feasibility study on the development of urban intelligent transportation network system in the era of big data," Communication World, vol. 27, no. 1, pp. 157-158, 2020.

[27] Y. Z. Liang, "System design of intelligent transportation data analysis platform under big data technology environment," Value Engineering, vol. 39, no. 15, pp. 243-244, 2020. 